\title{
Spatial Characteristics of Wideband Channels Using Leaky Coaxial Cables in Tunnel Scenario
}

\author{
Asad Saleem $\mathbb{D}$, Min Wang $\mathbb{D}$, Guoxin Zheng $\mathbb{D}$, and Xiaoyu Yin $\mathbb{D}$ \\ Key Laboratory of Specialty Fiber Optics and Optical Access Networks, Joint International Research Laboratory of \\ Specialty Fiber Optics and Advanced Communication, Shanghai Institute for Advanced Communication and Data Science, \\ Shanghai University, 200444, Shanghai, China
}

Correspondence should be addressed to Guoxin Zheng; gxzheng@staff.shu.edu.cn

Received 13 March 2019; Accepted 17 April 2019; Published 2 May 2019

Academic Editor: Sotirios K. Goudos

Copyright (C) 2019 Asad Saleem et al. This is an open access article distributed under the Creative Commons Attribution License, which permits unrestricted use, distribution, and reproduction in any medium, provided the original work is properly cited.

\begin{abstract}
This paper presents the spatial domain and propagation characteristics of different wideband channels using leaky coaxial cables (LCXs) for underground environment at $1.8 \mathrm{GHz}$ corresponding to the Long Term Evolution for Metro (LTE-M) standards. Angular dispersion of the signal significantly affects the channel capacity, and it can be exactly predicted in both the near and far fields for underground environments by means of a multimode waveguide. A ray tracing phenomenon was exploited to visualize the wave propagation for different transmitter and receiver antenna distances by using the time domain approach for both the horizontally and vertically polarized LCXs. In order to achieve this goal, we characterized the power azimuth spectrum (PAS) and power angle profile (PAP) for different tunnel dimensions and found that the PAS can be demonstrated by a zero-mean Gaussian distribution whose angular spread (AS) is dependent on the transmitter-receiver distances and the tunnel dimensions. The results demonstrate that the horizontally polarized LCX has higher angular spread values than the vertically polarized one, and the correlation coefficient follows the decreasing function with the increment of angular spread.
\end{abstract}

\section{Introduction}

The amount of data traffic has been increased explosively during the previous few years and the number of existing licensed spectrums are inadequate; Mobile Network Operators are considering the offloading of cellular network data to Wireless Local Area Networks (WLANs) as majority of the mobile devices endorse radio access [1]. The transmission packet drop and delay affect the performance of train control systems. The Communication Based Train Control (CBTC) network provides the prominent train location and continuous, outstanding, bidirectional data communications, by using ground safe equipment and vehicle equipment for underground scenario in order to fulfill the LTE-M standards [2-4]. The atmosphere in a tunnel is much more complicated and requires a careful analysis of the signal propagation. The diffractions and the reflections on the obstacles inside the railway tunnels cause even more serious multipath fading. To solve the unpredictable link problems induced through the multipath fading, the Multiple-Input Multiple-Output
(MIMO) systems has been recently used as a remarkable tool to improve the channel capacity of wireless radio channels $[5,6]$. Single-input single-output (SISO) channels for underground environment have been widely examined in terms of experiments and theory. Root mean square (RMS) delay spread, path losses, and capacity are the main emphasis of the SISO channels. The coherence bandwidth cuts down the increment of Tx-Rx separation, whereas the RMS delay spread increases with the increment of distance [7].

For an indoor environment such as railways and tunnel, leaky coaxial cables (LCXs) are extensively used as transmitting or receiving antennas for both side radio communications. LCXs provide stable and reliable signals. Normally, single LCX is used as one receiving or transmitting antenna. Therefore, more than one LCX is required to configure a MIMO system [8]. In linear service areas, a radiation characteristic through LCXs relies on the installation methods of LCXs, electromagnetic field intensity, and environmental specifications nearby the LCX [9]. Ray tracing approach is highly used to predict the radiated field and coupling losses 
in confined areas. In $[10,11]$, the authors used the ray tracing approach to estimate the distributive coupling losses and impulse response of the leaky waveguide, and it was found that the coupling loss decreases with the increment of slots period of leaky coaxial cable, and path loss decreases exponentially with the increase of separation distance between Tx and Rx. In [12], the radio signal attenuation measurements in concrete tunnels at four different frequencies $(0.455,0.915$, 2.45 , and $5.8 \mathrm{GHz}$ ) are studied. In this paper, a ray tracing (RT) method is used to model the propagation channel. The measuring data verifies the capability of the RT model to predict the radio frequency propagation. A modified radio frequency testing model that can be easily manufactured and used for radio propagation measurements in different tunnels is demonstrated in [13]. According to the proposed testing system, the radio frequency power attenuation with the distance in tunnel was measured at four different frequencies for both vertical and horizontal polarization schemes. Two analytical modeling approaches (the modal and the ray tracing) are practiced to model the radio frequency propagations in a tunnel environment. In [14], an extensive measurement campaign has been considered at $2.4 \mathrm{GHz}$ in Madrid subway in order to understand the precise characterization of a radio channel in tunnel atmosphere. Four different transmitters were installed in a $2 \mathrm{~km}$ long tunnel and the mobile receiver was located on the standard train. The propagation characteristics including path loss, near shadowing, shadow fading, level crossing rate, and fast fading have been computed and measured. The fifth generation $(5 \mathrm{G})$ mobile communication supports higher mobility until $500 \mathrm{~km} / \mathrm{h}$ which is essential for high speed trains. The spectrum provides the best enabler for the highly mobile users even when the wide channel sounding calibrations are not available and, moreover, the ray tracingbased algorithm was used to verify the proposed model [15].

In order to characterize the MIMO channel, the spatial domain becomes as essential as the temporal domain. The power azimuth spectrum (PAS) determines the spatial characteristics of the received signal power in the azimuthal direction. However, the angular spread (AS) is determined as the standard deviation of power azimuth spectrum, which is proportional to the RMS delay spread of power-delay-profile (PDP). Larger angular spread results in a smaller correlation coefficient. Angular spread predicts many characteristics of the MIMO channel such as the capacity and singular distribution. On the basis of the waveguide theory, it was revealed that the Gaussian distribution fits considerably well for the PAS [16, 17]. However, in literature a deficiency of PAS information in tunnel environment was observed. To the best of our knowledge, leaky coaxial cable based spatial characteristics in a real time tunnel scenario have not been reported yet.

Since there exists a substantial interest in the LTE-M channel in the tunnel scenario, we propose a radiated model for the spatial channels at $1.8 \mathrm{GHz}$. The combination of polarization and space diversity has been anticipated as an attractive approach to improve the spatial channel capacity. In this paper, we fill this gap by considering the joint polarization and space channel characteristics. We conducted a simulated campaign in the subway tunnels based on the ray tracing approach and further provided a comprehensive analysis for the anticipated results. The spatial correlation coefficients, power azimuth spectrum (PAS), power angle profile (PAP), and angular spread (AS) were explored for both the horizontally and vertically polarized LCXs in two different tunnels with different numbers of Tx and Rx antennas.

The rest of the paper is organized as follows. Section 2 deals with the radiated field based on theoretical approach for LCXs in tunnel. Section 3 defines the Nantong tunnel configurations. Section 4 demonstrates the PAS, the correlation coefficients, and the power angular profile (PAP) for Nantong and France central tunnels. Finally, Section 5 draws the conclusion of this paper.

\section{Radiated Field in Free Space}

Radiated field deals with the transfer of energy in the form of electric waves from one medium to another, and it follows the distance based inverse square law from the transmitter. Leaky coaxial cables based communication system is favorable for underground tunnels and mines because of its capability to work as both transmitting and receiving antennas. The cable is called leaky because of having gaps or slots on its outer conducting sheet which facilitate the emitting and receiving of electric signals in form of waves. Leaky cables are manufactured in different shapes and characteristics, based on the demand and surrounding situation, but here we discussed only two kinds of them, namely, horizontally polarized leaky cables and vertically polarized leaky cables. In vertically polarized leaky cables, slots of leaky cable are arranged vertically to cable propagation direction, and for horizontally polarized cables, the slots are set up horizontally to the propagation direction $[18,19]$. The outer and inner radius of leaky cable cylinder are assumed as b and a. The slot angle is considered as $2 a$, length of whole leaky cable is taken as $L$, and the width and length of slot are assumed as $w$ and $l$, respectively. The slot structure for horizontally and vertically polarized LCXs is given in Figure 1. Distance from fixed point $o$ to any point in free space is $r$ whose projection is $r^{\prime}$ in xoy coordinates. The leaky cable is positioned along $\mathrm{z}$-axis and the slots of the leaky cable are perpendicular from $\mathrm{z}$-axis. The slot width is slightly small so the radiated field is supposed to be constant. The circumferentially radiated field through leaky coaxial cable can be calculated as [20-22]

$$
E_{\varphi}=V(\theta, \varphi) \frac{e^{-j k_{0} r}}{r} \sin \theta
$$

where

$$
\begin{aligned}
& V(\theta, \varphi) \\
& =\frac{j V \cos \theta}{\pi^{2} b k_{0} \sin ^{3} \theta} \sum_{n=-5}^{n=+5} \frac{n e^{j n \varphi} j^{n+1}}{H_{n}^{(2)^{\prime}}\left(b k_{0} \sin \theta\right)} \\
& \quad \times\left(\frac{\sin n \alpha}{n}-\frac{m \sin m \alpha \cos n \alpha-n \cos m \alpha \sin n \alpha}{\left(m^{2}-n^{2}\right) \cos m \alpha}\right)
\end{aligned}
$$

where $m=k_{0} b, \varphi$ is the angle between $\mathrm{x}$-axis and $\mathrm{r}^{\prime}$, and $k_{0}$ is the wave number in free space, whereas $V=$ 


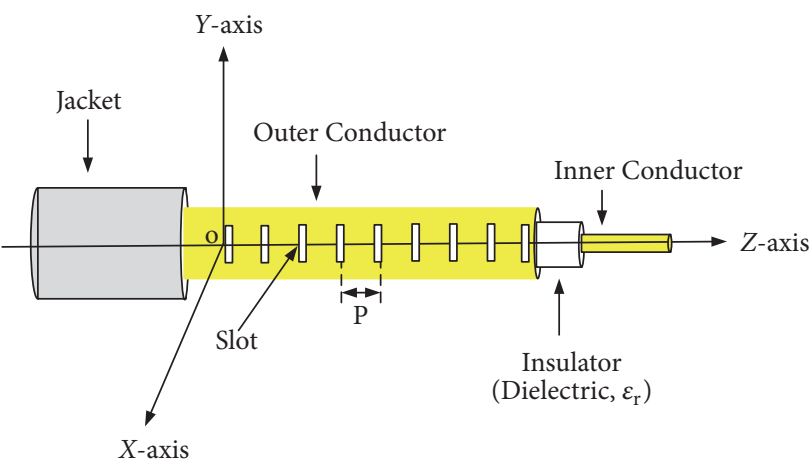

(a)

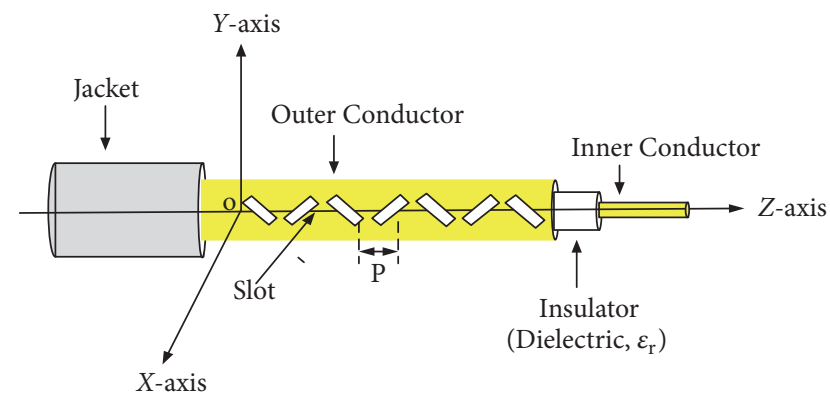

(b)

FIGURE 1: The slot structure of leaky coaxial cable for (a) horizontally polarized type of cable and (b) vertically polarized type of cable.

$\left(-j V_{0} \sqrt{\varepsilon_{r}}\right) /\left[2 k_{0} b \ln (b / a)\right], \varepsilon_{r}$ is the dielectric coefficient of dielectric layer of leaky cable, $H_{n}{ }^{(2)}{ }^{\prime}$ is the Hankel function of $n$th rank for the second type, $\omega$ is the angular frequency, $\epsilon_{0}$ is the free space dielectric coefficient, and $\theta$ is the angle among $\mathrm{z}$-axis and $\mathrm{r}$. By exploiting the ray tracing approach, the vertically polarized component $E_{y}$ of the circumferentially radiated field is given as [20]

$$
E_{y}=V\left(\theta_{i}, \varphi_{i}\right) \frac{e^{-j k_{0}\left(r_{i}+r_{i 1}\right)-j \beta_{i} P}}{r_{i}+r_{i 1}} \sin \theta_{i} \cos \varphi_{i} \Gamma_{h}
$$

where

$$
\begin{aligned}
& r_{i}=\sqrt{{x_{o}}^{2}+h_{0}^{2}+z_{0}^{2}} \\
& r_{i 1}=\sqrt{x_{0}^{2}+\left(h-h_{0}\right)^{2}+\left(i p-z_{0}\right)^{2}} \\
& \varphi_{i}=\arccos \left(\frac{x_{0}}{\sqrt{x_{0}^{2}+h_{0}^{2}}}\right), \\
& \theta_{i}=\arctan \left(\frac{\sqrt{{x_{0}}^{2}+h_{0}^{2}}}{z_{0}}\right) \\
& \beta=k_{0} \sqrt{\epsilon_{r}}, \\
& \gamma=\arctan \left(\frac{x_{0}}{\sqrt{z_{0}^{2}+h_{0}^{2}}}\right)
\end{aligned}
$$

where $\left(\epsilon_{1}-j \epsilon_{1}{ }^{\prime}\right)$ is the complex dielectric coefficient of the tunnel walls, $\Gamma_{h}$ is the reflection coefficient for horizontally polarized radiated signals, $i$ is the number of slots, $P$ is the period of LCX slots, $x_{0}$ is the horizontal distance between left metope of space and the cable, $h_{0}$ is the vertical distance between incident point and the cable, and $z_{0}$ is the longitudinal distance between incident point and the cable.
The reflection coefficient for a horizontally polarized radiated wave through LCX is specified below.

$$
\Gamma_{h}=\frac{\sin \gamma-\sqrt{\left(\epsilon_{1}-j \epsilon_{1}{ }^{\prime}\right)-\cos ^{2} \gamma}}{\sin \gamma+\sqrt{\left(\epsilon_{1}-j \epsilon_{1}{ }^{\prime}\right)-\cos ^{2} \gamma}}
$$

The reflection coefficient for the vertically polarized radiated wave is demonstrated as follows.

$$
\Gamma_{v}=\frac{\left(\epsilon_{1}-j \epsilon_{1}^{\prime}\right) \sin \gamma-\sqrt{\left(\epsilon_{1}-j \epsilon_{1}{ }^{\prime}\right)-\cos ^{2} \gamma}}{\left(\epsilon_{1}-j \epsilon_{1}^{\prime}\right) \sin \gamma+\sqrt{\left(\epsilon_{1}-j \epsilon_{1}^{\prime}\right)-\cos ^{2} \gamma}}
$$

The vertically polarized radiated field in response to all the slots of LCX can be estimated as follows.

$$
E_{y \Sigma}=\sum_{i=1}^{N} V\left(\theta_{i}, \varphi_{i}\right) \frac{e^{-j k_{0}\left(r_{i}+r_{i 1}\right)-j \beta_{i} P}}{r_{i}+r_{i 1}} \sin \theta_{i} \cos \varphi_{i} \Gamma_{v}
$$

\section{Setup Configuration}

The simulations are conducted based on ray tracing method in Nantong tunnel in Zhongtian Technology Company (ZTT). This tunnel is basically specifically used for radiated field measurements; ZTT-LCX of $50 \mathrm{~m}$ length was used for simulations and UHA9125D dipole antenna was used at the receiving side. Nantong tunnel is $100 \mathrm{~m}$ long and it is divided into two cross sections $[23,24]$. First $50 \mathrm{~m}$ long area consists of a rectangular tunnel of cross sectional area of $50 \mathrm{~m}$ (length) $\mathrm{x} 4.4 \mathrm{~m}$ (width) x $3 \mathrm{~m}$ (height), and the other $50 \mathrm{~m}$ tunnel consists of arched tunnel with cross section of $50 \mathrm{~m}$ (length) $\mathrm{x}$ $5 \mathrm{~m}$ (width) $\times 3 \mathrm{~m}$ (height). The tunnel walls are covered with reinforced concrete material and the tunnel wall material has the same characteristics as the real subway tunnel. There exist six locations for the LCX in tunnel along the wall, denoted as Loc-1 to Loc-6, but we selected Loc-3 for leaky cable. The Wireless Insite software was exploited for simulations in rectangular tunnel and it is commercially used for wave propagation for indoor environment [25]. The tunnel inner view based on the Wireless Insite software is given in Figure 2.

Leaky coaxial cable used as transmitting antenna was fixed at $1.9 \mathrm{~m}$ height from the ground, and $\mathrm{Rx}$ antenna was 


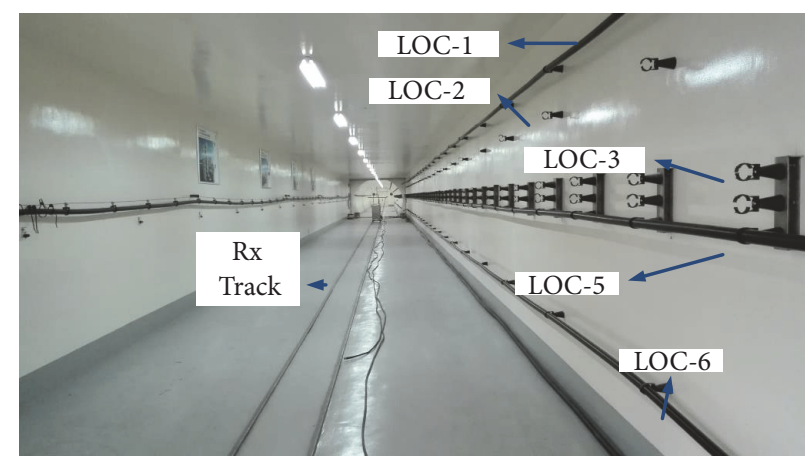

(a)

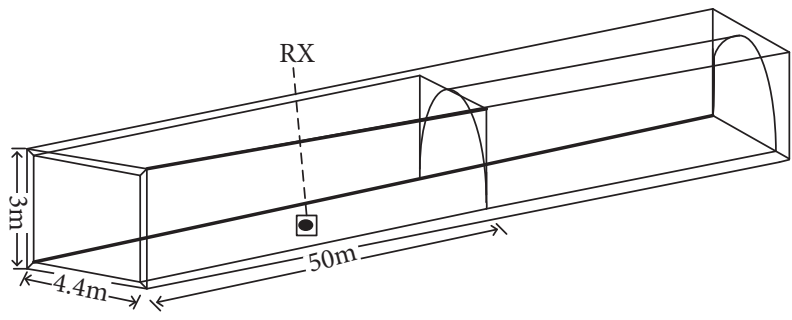

(b)

FIGURE 2: The Nantong tunnel: (a) actual inner view, (b) construction of Nantong tunnel in Wireless Insite software.

mounted at $1.6 \mathrm{~m}$ height from the ground. LCX cable was laid along $\mathrm{z}$-axis and the total height of tunnel along $\mathrm{y}$ axis is $3 \mathrm{~m}$. The minimum distance between $\mathrm{Tx}$ and $\mathrm{Rx}$ antennas was calculated as $2 \mathrm{~m}$ along $\mathrm{x}$-axis. Horizontally and vertically polarized LCXs were used for simulations based on ray tracing approach. The transmitted power was adjusted as $20 \mathrm{dBm}$ and sampling rate was considered as $81.6 \mathrm{MHz}$. The LCX slots period was taken as $0.147 \mathrm{~m}$ and the effective bandwidth was set to $40.8 \mathrm{MHz}$ with a carrier frequency of $1.8 \mathrm{GHz}$. The antennas gain was fixed as $2.15 \mathrm{dBi}$ and 8 numbers of reflections were perceived. The radii of outer and inner conductors of LCX are fixed as $16 \mathrm{~mm}$ and $6 \mathrm{~mm}$, respectively. Initially, the middle of the tunnel location was used for simulations based on ray tracing method and W.Insite software for Rx antenna. The Rx antenna was located at $25 \mathrm{~m}$ away from the entrance of rectangular tunnel. The operating frequency was set as $1.8 \mathrm{GHz}$ because it provides strong and steady LTE coverage for tunnel environment.

\section{Results and Analysis}

In order to understand the LCX based spatial channel characteristics in Nantong tunnel, we considered three properties, namely, power azimuth spectrum, power angular profile, and channel correlation coefficient.

4.1. Power Azimuth Spectrum (PAS). To visualize the PAS in the tunnel, the corresponding power and angles need to be estimated based on the operating frequency $(1.8 \mathrm{GHz})$ and tunnel dimensions. The truncated Gaussian distribution is observed to be the suitable fit for the measured PAS. By using the nonlinear least square distribution fitting, the PAS for the vertically and horizontally polarized LCXs with different receiving distances can be demonstrated as follows [26, 27]:

$$
P(\varphi)=\frac{Q}{\sqrt{2 \pi \sigma^{2}}} e^{\left(-1 / 2 \sigma^{2}\right)\left(\varphi-\varphi_{0}\right)^{2}}
$$

where $\varphi$ is the azimuth angle, $-\Delta \varphi+\varphi_{0} \leq \varphi \leq \Delta \varphi+\varphi_{0}$ and $\Delta \varphi$ is uniformly distributed along $-\pi$ to $\pi, \sigma$ is the angular spread, and $\varphi_{0}$ is the mean angle of arrival (AoA). For simplicity we considered $\Delta \varphi=\pi$. The normalization factor $Q$ can be calculated as

$$
Q=\frac{1}{\operatorname{erf}\left(\Delta \varphi / \sqrt{2 \sigma^{2}}\right)}
$$

where erf(.) illustrates the error function. The Gaussian distribution fitting for PAS was achieved through the mean AoA of $0^{\circ}$ (line of sight direction) for the receivers $(R x=25 \mathrm{~m})$, based on the ray tracing software (W.Insite) for horizontally and vertically polarized LCXs, and further these simulation results are compared with the analytical results by using (7) and (8) as shown in Figures 3(a) and 3(b). The analytical results were estimated by using the MATLAB software. The horizontal polarization based simulation shows better performance in terms of AS than the vertically polarized cables. The power distribution turns wider for the larger angle as angular spread increases, which further causes decrement in the spatial correlation coefficients under the same antenna spacing conditions. Specifically, when angular spread of power azimuth spectrum is smaller, many rays which comprise each path fall at each antenna with almost the same angle, which indicates that these rays are correlated to each other; however, the magnitude of some signals is almost similar, whereas their phases are not similar from their angle of arrivals. Since the channel capacity reduces as the correlation among the antenna elements increases, antenna spacing must be set as large enough in order to decrease the correlation. There exists a metal box in start of tunnel which causes higher number of reflections, so the starting area of tunnel has less angular spread. In arched tunnel, one moveable metallic trolley is placed, which has the same characteristics as the real time vehicle. At the junction of the rectangular and arched tunnel, some additional concrete wall portion is found, and there is also a metal door at the end of tunnel; due to these constraints, $\mathrm{Rx}$ at middle part of tunnel has comparatively higher angular spread.

LCXs are regarded as scattered antenna arrays; the Rx gets the radiated signals from entire associated slots of the leaky cable, and then it further transfers them through the subchannels. Therefore, the distribution of AoA is determined by the response of all subchannels. The various radiated signals directions enlarge the AS value, which is apparently suitable 


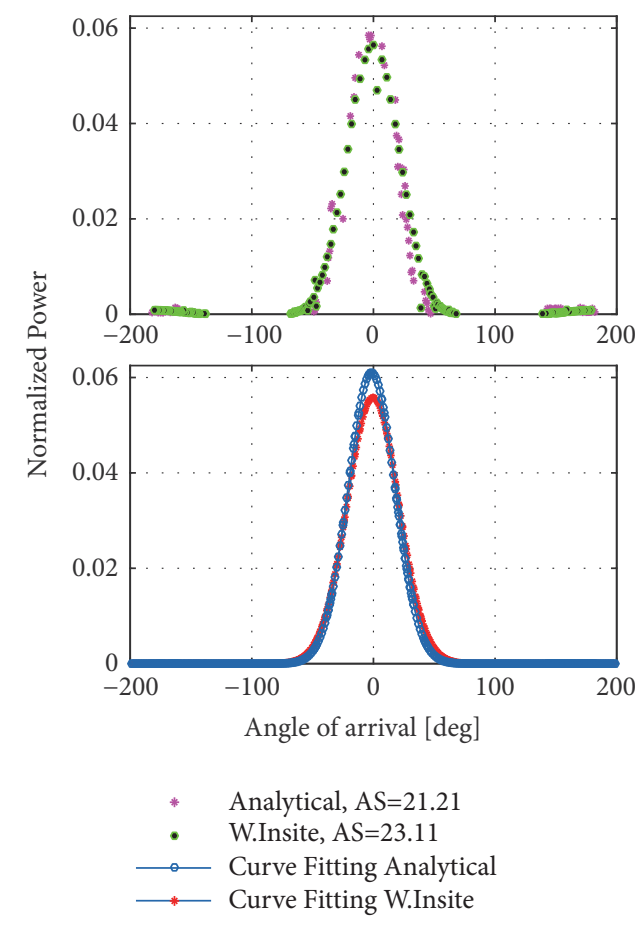

(a)

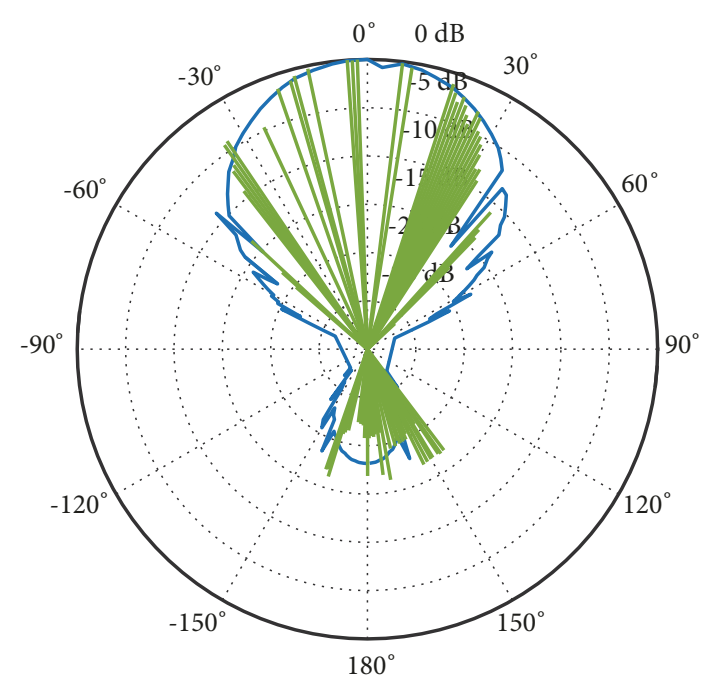

---- Analytical

----- W.Insite

(c)
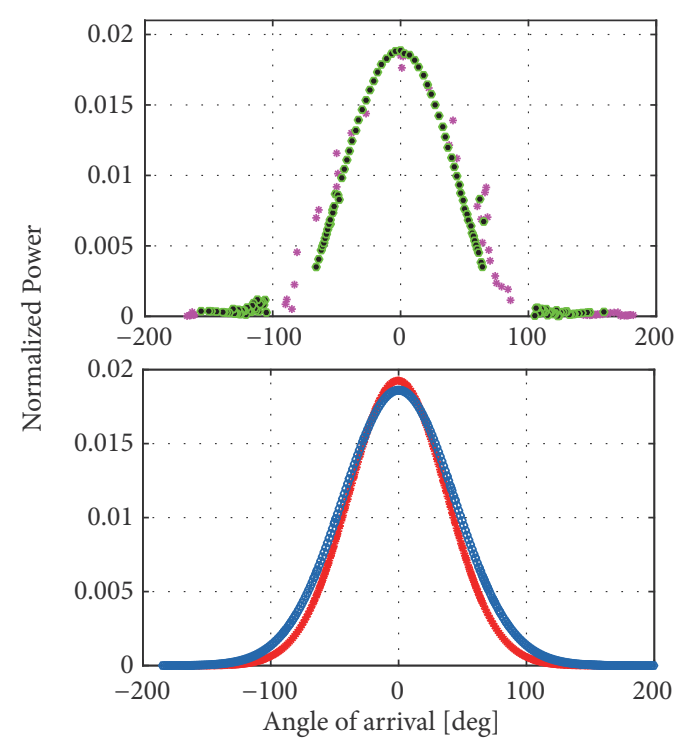

* Analytical, AS $=39.15$

- W.Insite, $\mathrm{AS}=42.63$

$\ldots$ Curve Fitting Analytical

— Curve Fitting W.Insite

(b)

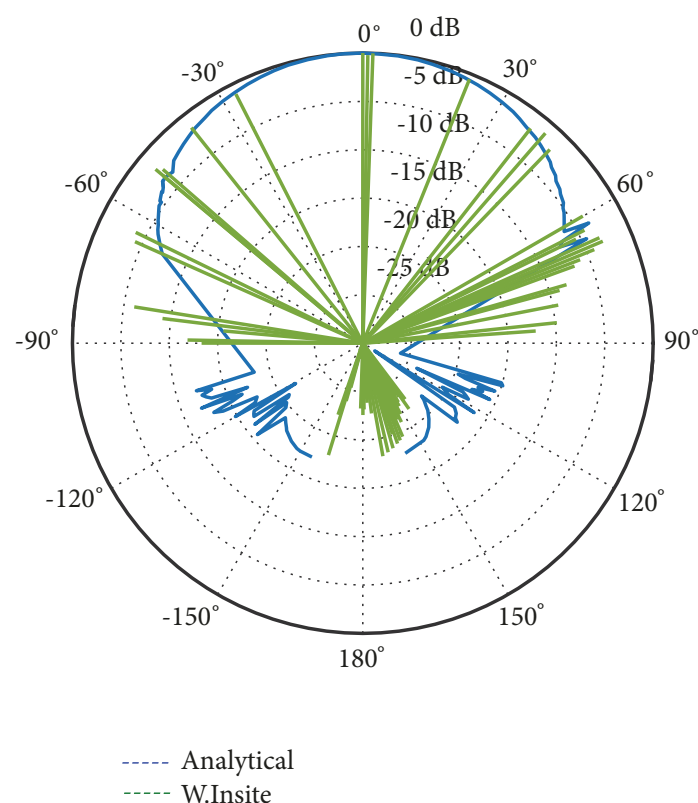

(d)

FIGURE 3: The PAS characterization for Nantong tunnel based on analytical results and ray tracing software (W.Insite): (a) vertically polarized LCX with Rx at $25 \mathrm{~m}$ away from entrance, (b) horizontally polarized LCX with Rx at $25 \mathrm{~m}$. The PAP characteristics for Nantong tunnel based on analytical results and ray tracing software (W.Insite): (c) vertically polarized LCX with Rx at $25 \mathrm{~m}$, (d) horizontally polarized LCX with Rx at $25 \mathrm{~m}$.

to provide the decorrelation of spatial channels $[28,29]$. For horizontal polarization, the incident rays at the tunnel ceiling and floor are horizontally polarized but vertical polarization is observed at the tunnel wall. The converse case is considered for the vertically polarized rays. The height of tunnel is shorter than its width, and thus the reflected signals from tunnel wall have larger AoA as compared to the tunnel ceiling and floor. The vertically polarized coefficients increase according to the incidence angles. However, a horizontally polarized coefficient has a Brewster angle and it is always smaller than that of the vertically polarized coefficient, which further causes the greater AS for horizontally polarized LCX 
TABLE 1: AS values for Nantong tunnel.

\begin{tabular}{lcc}
\hline Polarization Type & Rx Antenna Distance & AS value \\
\hline Vertical & $1 \mathrm{~m}$ & 18.18 \\
Vertical & $49 \mathrm{~m}$ & 17.44 \\
Horizontal & $1 \mathrm{~m}$ & 37.03 \\
Horizontal & $49 \mathrm{~m}$ & 32.06 \\
\hline
\end{tabular}

as compared to the vertically polarized cable. We have further estimated the AS values for receivers at $1 \mathrm{~m}$ and $49 \mathrm{~m}$ away from the entrance of Nantong tunnel and the values are illustrated in Table 1. From the results, it can be seen that AS value is higher in the middle of the tunnel because of relatively less correlation.

4.2. Power Angle Profile and Correlation Coefficients. The PAP comparison for simulation and analytical results for horizontally and vertically polarized LCXs for different Tx$\mathrm{Rx}$ distances are normalized by the maximum power components (MPC). The $0 \mathrm{~dB}$ power is regarded as the maximum power for each case. Due to small metal box which is placed closer to the sidewalls, there are slight differences between simulated and analytical results in tunnel. The PAP for $\mathrm{Rx}$ at $25 \mathrm{~m}$ away from start of tunnel is given in Figures 3(c) and $3(\mathrm{~d})$.

As we notice in Figure 3, the shapes for all PAPs are affected significantly due to the physical presence of metallic box, some metallic trolley, metallic door, and extra concrete wall, since the highest number of reflections originates from the metal door. Afterward, at receiving antenna location, the AoA computation factors were estimated through characterizing the multipath power. The maximum AoA delivers the directions of all multipath components regarding the highest power. The concrete wall and metal door which have higher reflective surfaces substantially increase the number of multipath reflections through the receivers. For the antenna elements separated by distance $\mathrm{d}$, the normalized antenna separation $D=2 \pi d / \lambda$, and when the mean AoA is $\varphi_{0}$, the spatial correlation coefficients of received signals can be written as follows $[30,31]$ :

$$
\rho_{c}(D)=R_{X X}(D)+j R_{X Y}(D)
$$

where

$$
\begin{aligned}
& R_{X X}(D)=J_{0}(D)+Q \sum_{m=1}^{\infty} J_{2 m}(D) e^{-2 \sigma^{2} m^{2}} \cos \left(2 m \varphi_{0}\right) \\
& . \operatorname{Re}\left[\operatorname{erf}\left(\frac{\Delta \varphi}{\sigma \sqrt{2}}-j m \sigma \sqrt{2}\right)\right. \\
& \left.\quad-\operatorname{erf}\left(-\frac{\Delta \varphi}{\sigma \sqrt{2}}-j m \sigma \sqrt{2}\right)\right]
\end{aligned}
$$

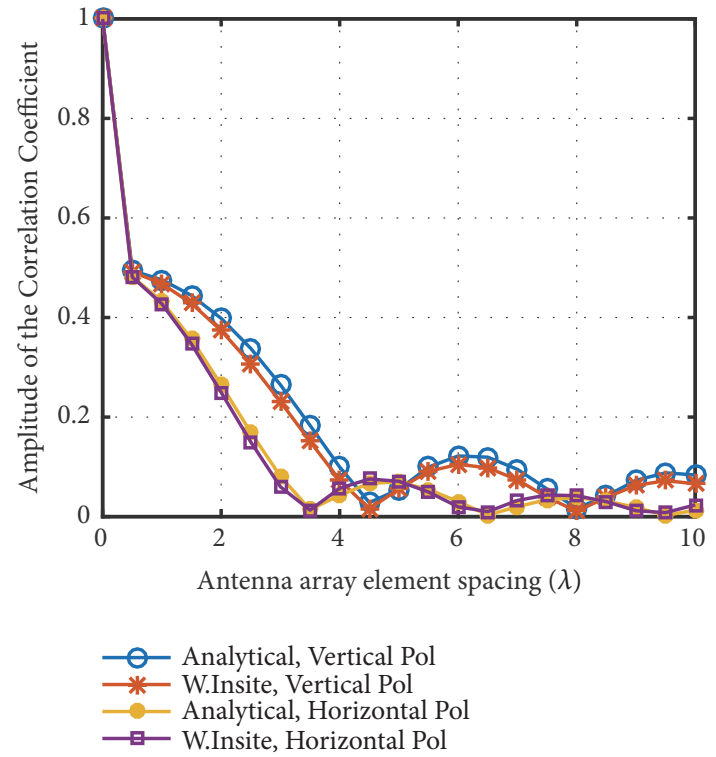

FIGURE 4: The correlation coefficient for horizontally and vertically polarized LCX.

and

$$
\begin{array}{r}
R_{X Y}(D) \\
=Q \sum_{m=1}^{\infty} J_{2 m+1}(D) e^{-2 \sigma^{2}(m+1 / 2)^{2}} \sin \left((2 m+1) \varphi_{0}\right) \\
. \operatorname{Re}\left[\operatorname{erf}\left(\frac{\Delta \varphi}{\sigma \sqrt{2}}-j \sigma \sqrt{2}\left(m+\frac{1}{2}\right)\right)\right. \\
\left.\quad-\operatorname{erf}\left(-\frac{\Delta \varphi}{\sigma \sqrt{2}}-j \sigma \sqrt{2}\right)\left(m+\frac{1}{2}\right)\right]
\end{array}
$$

where $J_{m}$ (.) is the mth order Bessel function of first kind. Here, we considered the $\mathrm{Rx}$ at $25 \mathrm{~m}$ distances from entrance in rectangular Nantong tunnel. The joint spatial correlation coefficients for $50 \mathrm{~m}$ long vertically and horizontally polarized LCXs are given in Figure 4. The results show good agreement between simulated and analytical results. We can also notice that the spatial correlation coefficients reduce to nearly zero at $3.5 \lambda$ and $4.5 \lambda$ when the angular spreads are estimated as 39.15 and 21.21 , respectively, for horizontally and vertically polarized leaky coaxial cables. Therefore, the separation distance should be adjusted to be approximately larger than $3.5 \lambda$ for horizontally polarized LCXs. The spatial 
TABLE 2: AS values in France central tunnel.

\begin{tabular}{lcc}
\hline Polarization Type & Rx Antenna Distance & AS value \\
\hline Horizontal & $40 \mathrm{~m}$ & 38.87 \\
Horizontal & $60 \mathrm{~m}$ & 36.99 \\
Horizontal & $100 \mathrm{~m}$ & 31.43 \\
Vertical & $40 \mathrm{~m}$ & 31.86 \\
Vertical & $60 \mathrm{~m}$ & 27.80 \\
Vertical & $100 \mathrm{~m}$ & 25.63 \\
\hline
\end{tabular}

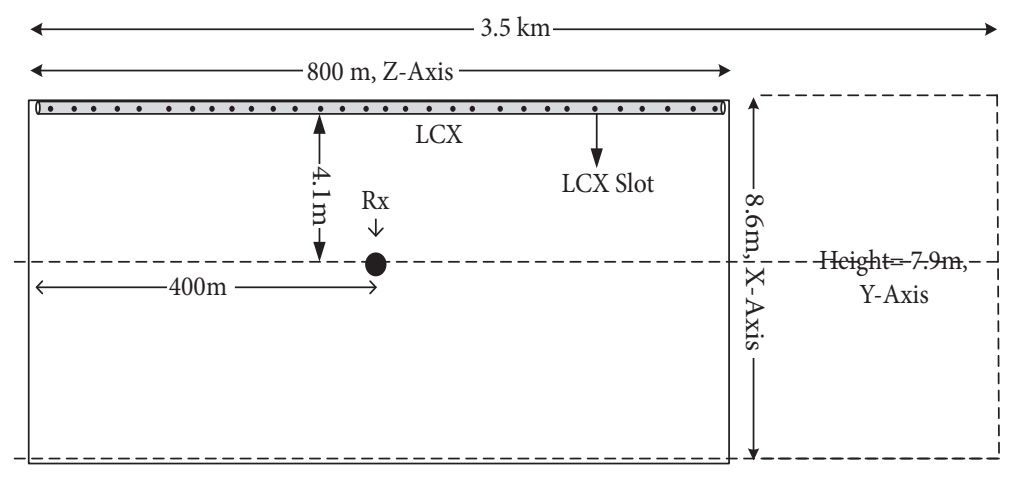

FIGURE 5: The France central tunnel sketch.

correlation coefficient decreases with the increment of AS for all these three receivers.

4.3. Massif Central Tunnel. For further investigation of spatial channel characteristics, the simulated approach for Massif central tunnel in south-central France was considered to investigate the PAS, PAP, and spatial correlation coefficients [32-34]. This tunnel has a cross sectional area of $3.5 \mathrm{~km}$ (length) $\times 18.6 \mathrm{~m}$ (width) $\times 1.9 \mathrm{~m}$ (height), and the walls of this tunnel are covered with the concrete material as shown in Figure 5. This tunnel is also used for analyzing the propagation characteristics.

We considered the $800 \mathrm{~m}$ long LCX for our experiments along $\mathrm{z}$-axis with the period of slots as $0.6 \mathrm{~m}$, and the $\mathrm{Rx}$ was placed $4.1 \mathrm{~m}$ away from LCX along $\mathrm{x}$-axis. The distance of the $\mathrm{Rx}$ from entrance was fixed as $400 \mathrm{~m}$ away (middle of LCX). The heights of $\mathrm{Rx}$ and Tx antennas were $1.6 \mathrm{~m}$ and $1.9 \mathrm{~m}$, respectively, along $y$-axis. The UHA9125D dipole antenna was used as Rx antenna, and the other parameters are the same as those given earlier for Nantong tunnel. The angular spread and PAP were calculated for both horizontally and vertically polarized cables by using (8) and (10) as given in Figures 6(a), 6(b), 6(c), and 6(d).

From Figure 6, it is obvious that the Gaussian distribution fitting of spatial channel for the horizontally polarized cables has better performance, and the analytical and simulated results have good agreement. The horizontally polarized cable has higher angular spread than the other. Due to the increment in AS, the power distribution becomes wider and, as a result, the correlation coefficients values decrease under the same antenna spacing. Because of the height of tunnel which is smaller than its width, higher AS values were achieved here. From the reflection coefficients point of view, the power of reflected rays at the tunnel wall has higher magnitude for the horizontal polarization than the vertical polarization. We further computed the AS at different distances of Rx antennas such as $40 \mathrm{~m}, 60 \mathrm{~m}$, and $100 \mathrm{~m}$ from the start of central tunnel, and the results are demonstrated in Table 2. The AS decreases with the increment of distance of $\mathrm{Rx}$ antennas due to the increment of correlation. Afterward, the spatial correlation coefficients were calculated for the receiver at $400 \mathrm{~m}$ away from entrance in Massif central tunnel as depicted in Figure 7. The results demonstrate that correlation coefficients for horizontally polarized LCX cable have lower value than the vertically polarized cable, and we found that correlation coefficient values reduce to nearly zero at 2.9 $\lambda$ and $3.4 \lambda$ for horizontally and vertically polarized leaky cables, respectively. The spatial correlation among antenna elements basically depends on the PAS, mean AoA, and antenna spacing. Therefore, the spacing between antennas must be set large enough to reduce the correlation. As the horizontally polarized scenario has higher AS value than the vertically polarized one in Massif central tunnel, it can be seen from Figure 7 that the spatial correlation coefficient decreases with the increment of angular spread even for the same antennas spacing.

By analyzing Figures 3, 4, 6, and 7, it can be concluded that length of LCX, the separating distance between antennas, and the tunnel environment have great influence on the spatial characteristics. An efficient performance can be achieved when these parameters are arranged properly and this is essential for the reliable communication in underground scenarios. Our analysis also showed that horizontally polarized LCX have better performance than the vertically polarized cable for Nantong and France central tunnels. 

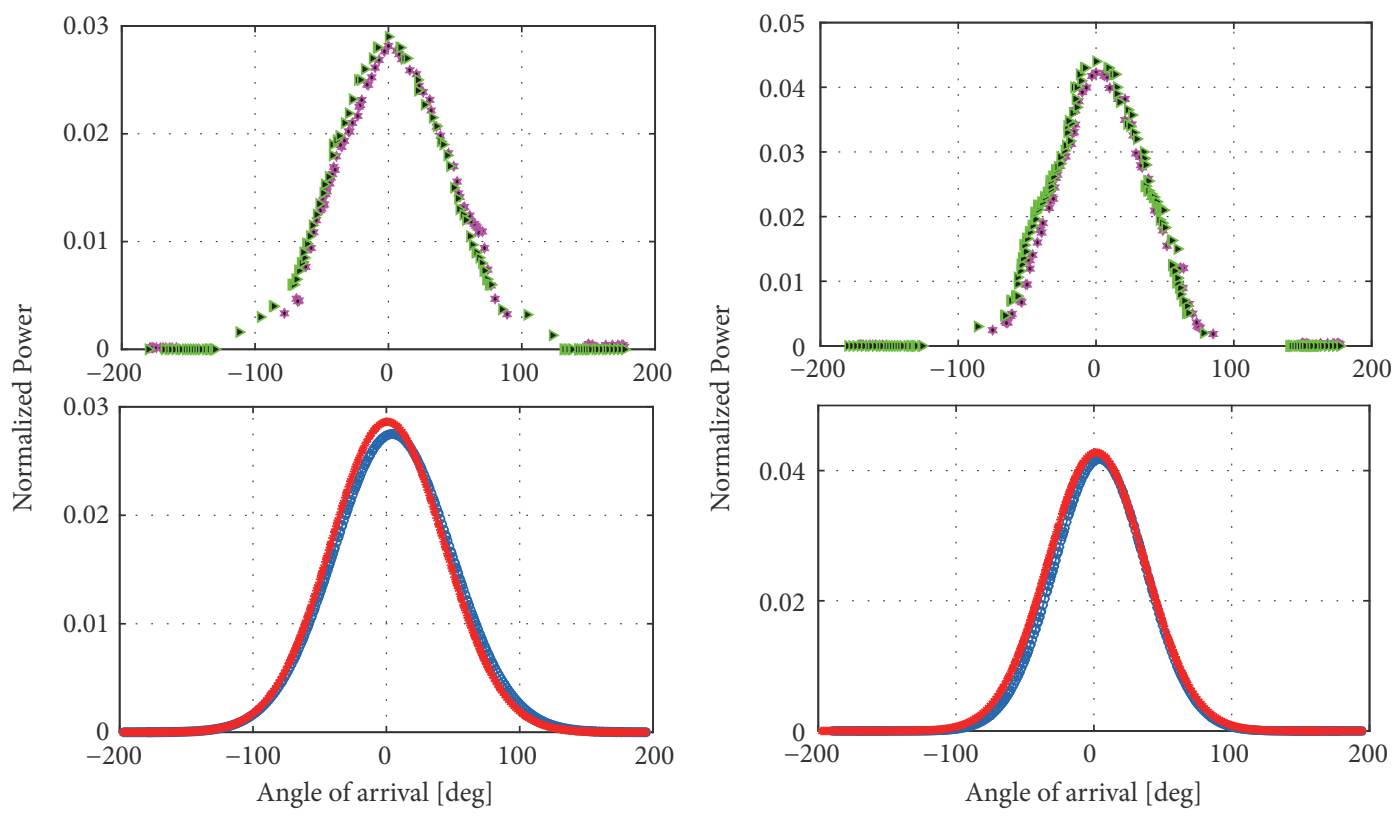

* Analytical, AS $=43.97$

- W.Insite, $\mathrm{AS}=42.60$

- Analytical, Vertical Pol

Curve Fitting, W.Insite

(a)

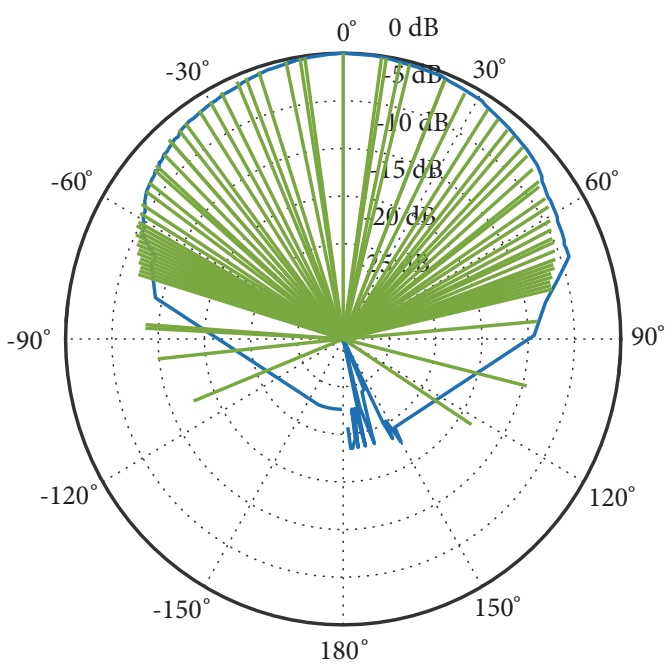

* Analytical, AS=34.23

* W.Insite, AS=35.16

$\longrightarrow$ Curve Fitting, Analytical

$\because$ Curve Fitting, W.Insite

(b)

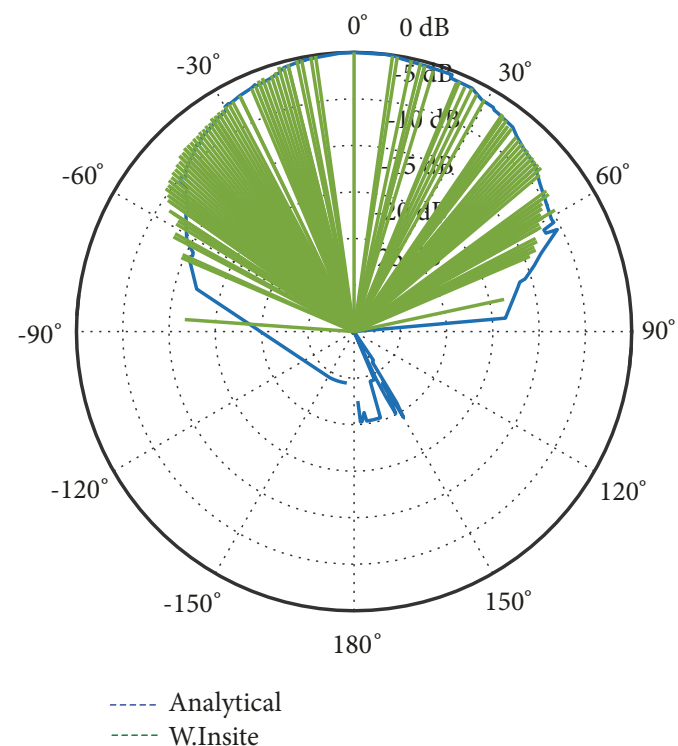

(d)

FIgURE 6: The PAS characterization for France central tunnel based on analytical results and ray tracing software (W.Insite): (a) horizontally polarized LCX with Rx at $400 \mathrm{~m}$ away from entrance, (b) vertically polarized LCX with Rx at $400 \mathrm{~m}$. The PAP characteristics for France central tunnel based on analytical results and ray tracing software (W.Insite): (c) horizontally polarized LCX with Rx at $400 \mathrm{~m}$, (d) vertically polarized LCX with Rx at $400 \mathrm{~m}$.

\section{Conclusion}

This paper investigates the LCX based spatial characteristics of a radio channel in the tunnel environment by using the radiated field and ray tracing method at $1.8 \mathrm{GHz}$. In order to investigate the spatial channel, initially the simulation campaign based on the Wireless Insite software was used for Nantong tunnel and France central tunnel. The receivers were placed in start $(R x=1 m)$, middle $(R x=25 m)$, and end $(\mathrm{Rx}=49 \mathrm{~m})$ of Nantong tunnel and at $400 \mathrm{~m}$ away from 

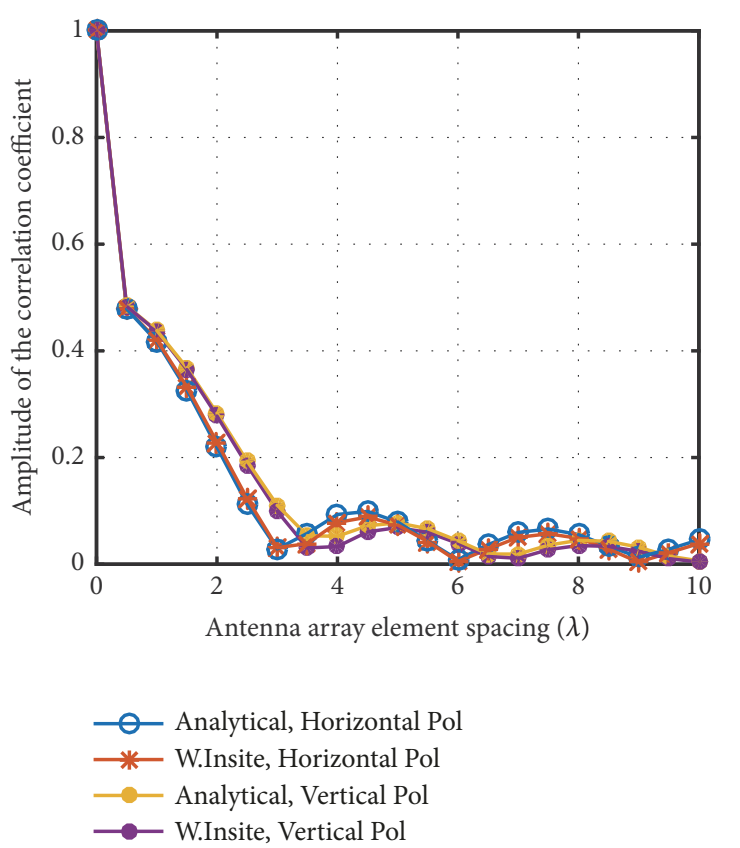

FIGURE 7: The correlation coefficient for horizontally and vertically polarized LCX for France central tunnel.

entrance in France central tunnel. For better understanding of this work, the main contributions are summarized as follows:

(1) The PAS for both polarization configurations is precisely demonstrated through the truncated Gaussian distribution function.

(2) We perceived that for receiver in the middle (25m) of Nantong rectangular tunnel, the power azimuth spectrum has higher angular spread than the other two receivers.

(3) We further investigated the PAP for both the horizontally and vertically polarized cables. It is concluded that the horizontally polarized cables have higher AS than the vertically polarized LCX in tunnel environment.

(4) Additionally, we found that the spatial correlation coefficient decreases with the increment of angular spread even for the same antennas spacing in both tunnels.

(5) As a result, this paper delivers a proficient model for the optimal system design, and it conveys an efficient direction for the LTE-M channel through consideration of the LCXs deployment in the tunnel scenario.

\section{Data Availability}

The analytical and simulated data used to support the findings of this study have not been made available because of the protection of intellectual property rights. Moreover, the data is shared with our partner company and will be used in our future work.

\section{Conflicts of Interest}

The authors declare that they have no conflicts of interest.

\section{Acknowledgments}

This work was supported by the National Natural Science Foundation of China under Grant 61571282 and 61871261.

\section{References}

[1] J. Wang and V. C. M. Leung, "An incentive engineering mechanism for optimizing handoff decisions between cellular data networks and WLANs," in Proceedings of the NOMS 2008 IEEE/IFIP Network Operations and Management Symposium: Pervasive Management for Ubiquitous Networks and Services, pp. 783-786, Brazil, April 2008.

[2] G. Zheng, Y. Ruan, and K. Kai, “The channel emulator for CBTC wireless communication system," in Proceedings of the 20107 th International Symposium on Communication Systems, Networks and Digital Signal Processing, CSNDSP 2010, pp. 464-468, UK, July 2010.

[3] H. Wang, F. R. Yu, L. Zhu, T. Tang, and B. Ning, "Finite-state Markov modeling for wireless channels in tunnel communication-based train control systems," IEEE Transactions on Intelligent Transportation Systems, vol. 15, no. 3, pp. 1083-1090, 2014.

[4] X. Wang, L. Liu, T. Tang, and W. Sun, "Enhancing Communication-Based Train Control Systems Through Train-to-Train Communications," IEEE Transactions on Intelligent Transportation Systems, no. 99, pp. 1-8, 2018.

[5] Z. Sun, I. F. Akyildiz, and G. P. Hancke, "Capacity and outage analysis of MIMO and cooperative communication systems in underground tunnels," IEEE Transactions on Wireless Communications, vol. 10, no. 11, pp. 3793-3803, 2011.

[6] A. E. Forooshani, A. A. Lotfi-Neyestanak, and D. G. Michelson, "Optimization of antenna placement in distributed mimo systems for underground mines," IEEE Transactions on Wireless Communications, vol. 13, no. 9, pp. 4685-4692, 2014.

[7] H. M. El-Azhari, M. Nedil, I. Ben Mabrouk, and L. Talbi, "Offbody channel characterization at $2.45 \mathrm{GHz}$ in underground mine environment," in Proceedings of the 2014 IEEE Antennas and Propagation Society International Symposium, APSURSI 2014, pp. 251-252, USA, July 2014.

[8] T. Higashino and M. Okada, "A wireless sensing technique based on channel estimation in leaky coaxial cable antenna system," in Proceedings of the 31st General Assembly and Scientific Symposium of the International Union of Radio Science, URSI GASS 2014, pp. 1-4, China, August 2014.

[9] C. Zhang, J. Wang, M. Chen, and Z. Zhang, "Radiation characteristic of the leaky circular waveguide with periodic slots," IEEE Antennas and Wireless Propagation Letters, vol. 11, pp. 503-506, 2012.

[10] X. Huo, D. Li, X. Han, and J. Wang, "Effects of structural and environmental parameters on the coupling loss of leaky rectangular waveguide in tunnel," in Proceedings of the Loughborough Antennas and Propagation Conference, LAPC 2015, pp. 1-4, Loughborough, UK, November 2015.

[11] Z. Yun and M. F. Iskander, "Ray tracing for radio propagation modeling: Principles and applications," IEEE Access, vol. 3, pp. 1089-1100, 2015. 
[12] Z. Chenming and R. Jacksha, "Modeling and measurement of radio propagation in tunnel environments," IEEE Antennas and Wireless Propagation Letters, vol. 16, pp. 1431-1434, 2017.

[13] C. Zhou, J. Waynert, T. Plass, and R. Jacksha, "Modeling RF propagation in tunnels," in Proceedings of the 2013 IEEE Antennas and Propagation Society International Symposium, APSURSI 2013, pp. 1916-1917, USA, July 2013.

[14] K. Guan, Z. D. Zhong, J. I. Alonso, and C. Briso-Rodríguez, "Measurement of distributed antenna systems at $2.4 \mathrm{GHz}$ in a realistic subway tunnel environment," IEEE Transactions on Vehicular Technology, vol. 61, no. 2, pp. 834-837, 2012.

[15] K. Guan, B. Ai, B. Peng et al., "Towards realistic high-speed train channels at 5G millimeter-wave band - Part I: Paradigm, significance analysis, and scenario reconstruction," IEEE Transactions on Vehicular Technology, vol. 67, no. 10, pp. 9112-9128, 2018.

[16] A. E. Forooshani, R. D. White, and D. G. Michelson, "Effect of antenna array properties on multipleinput-multiple-output system performance in an underground mine," IET Microwaves, Antennas \& Propagation, vol. 7, no. 13, pp. 1035-1044, 2013.

[17] R. Sun, D. W. Matolak, C. Tao, L. Liu, Z. Tan, and T. Zhou, "Investigation of MIMO channel characteristics in a twosection tunnel at $1.4725 \mathrm{GHz}$," International Journal of Antennas and Propagation, vol. 2017, Article ID 3693149, 12 pages, 2017.

[18] Z. Zixin, Y. Xiaodong, and G. Shuxiang, "A coupling loss algorithm of leaky coaxial cable in the blind zone," in Proceedings of the 2008 IEEE International Conference on Mechatronics and Automation, ICMA 2008, pp. 919-923, Japan, August 2008.

[19] F. Sayadi, M. Ismail, N. Misran, and K. Jumari, "Radio coverage inside tunnel utilizing leaky coaxial cable base station," Journal of Applied Sciences, vol. 9, no. 16, pp. 2887-2896, 2009.

[20] X. Zhang, X. Yang, L. Guo, and Z. Zhao, "Research on the radiated field's distribution of leaky coaxial cable in the blind zone," in Proceedings of the 1st International Symposium on Systems and Control in Aerospace and Astronautics, pp. 575-578, China, January 2006.

[21] D. Li and J. Wang, "Effect of antenna parameters on the field coverage in tunnel environments," International Journal of Antennas and Propagation, vol. 2016, Article ID 8180124, 10 pages, 2016.

[22] M. Piette, S. Collin, R. VanThienen, and P. Delogne, "Electromagnetic field produced by a pair of parallel leaky feeders in a tunnel," in Proceedings of the Progress in Electromagnetic Research Symposium, pp. 313-316, 2004.

[23] Y. Wu, G. Zheng, A. Saleem, and Y. P. Zhang, "An experimental study of MIMO performance using leaky coaxial cables in a tunnel," IEEE Antennas and Wireless Propagation Letters, vol. 16, pp. 1663-1666, 2017.

[24] Y.-T. Pan, G.-X. Zheng, and C. Oestges, "Characterization of polarized radio channel with leaky coaxial cable in a tunnel-like environment," IEEE Antennas and Wireless Propagation Letters, vol. 16, pp. 2803-2807, 2017.

[25] Wireless InSite User's Manual, Remcom Incorporated, State College PA, USA, 2011.

[26] J. Zhang, D. Dong, X. Nie et al., "Propagation characteristics of wideband relay channels in urban environment," in Proceedings of the 2009 4th International Conference on Communications and Networking in China, CHINACOM 2009, pp. 1-6, China, August 2009.

[27] Y. S. Cho, J. Kim, W. Y. Yang, and C. G. Kang, MIMO-OFDM Wireless Communications with MATLAB, John Wiley \& Sons, 2010.
[28] F. Pérez Fontán and P. Mariño Espiñeira, Modeling the Wireless Propagation Channel: A Simulation Approach with MATLAB, vol. 14 of Wiley Series on Wireless Communications And Mobilecomputing, 2008.

[29] N. Moraitis, P. Constantinou, and D. Vouyioukas, "Power angle profile measurements and capacity evaluation of a SIMO system at $60 \mathrm{GHz}$," in Proceedings of the 2010 IEEE 21st International Symposium on Personal Indoor and Mobile Radio Communications, PIMRC 2010, pp. 1027-1031, Turkey, September 2010.

[30] L. Schumacher, K. I. Pedersen, and P. E. Mogensen, "From antenna spacings to theoretical capacities - guidelines for simulating MIMO systems," in Proceedings of the 13th IEEE International Symposium on Personal, Indoor and Mobile Radio Communications, PIMRC 2002, vol. 2, pp. 587-592, Portugal, September 2002.

[31] C. Ziółkowski and J. M. Kelner, "Estimation of the reception angle distribution based on the power delay spectrum or profile," International Journal of Antennas and Propagation, vol. 2015, Article ID 936406, 14 pages, 2015.

[32] Z. Xingqi and C. D. Sarris, "A Gaussian beam approach for embedding antennas into vector parabolic equation based propagation models," in Proceedings of the 2016 IEEE International Symposium on Antennas and Propagation (APSURSI), pp. 21052106, 2016.

[33] D. G. Dudley, M. Liénard, S. F. Mahmoud, and P. Degauque, "Wireless propagation in tunnels," IEEE Antennas and Propagation Magazine, vol. 49, no. 2, pp. 11-26, 2007.

[34] X. He, D. Li, J. Wang, and S. Li, "A new ADI-PE scheme with fourth-order accuracy for radio wave propagation prediction in tunnels," in Proceedings of the 2014 International Symposium on Antennas and Propagation, ISAP 2014, pp. 259-260, Taiwan, December 2014. 


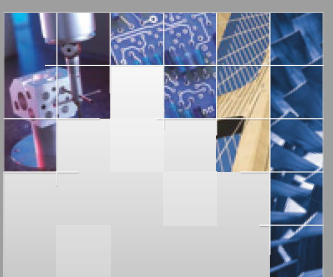

\section{Enfincering}
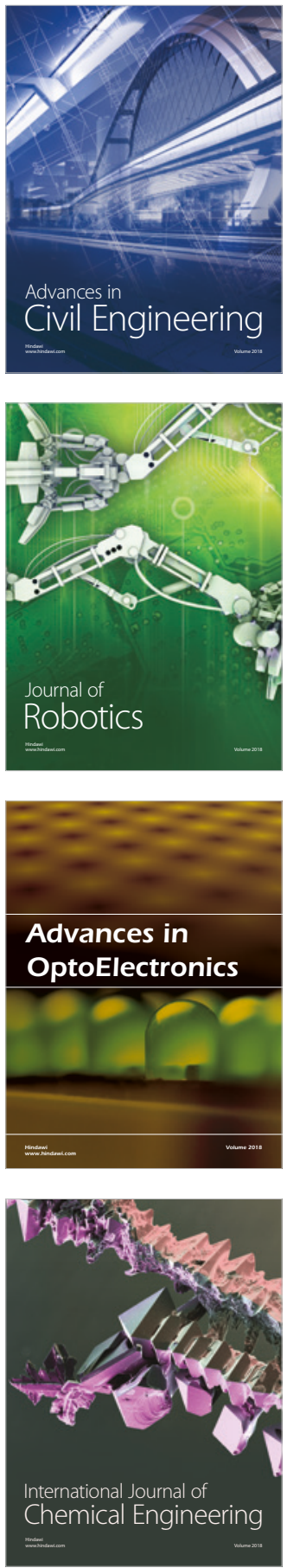

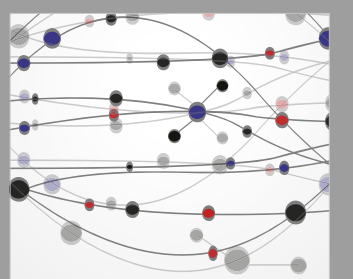

\section{Rotating \\ Machinery}

The Scientific World Journal

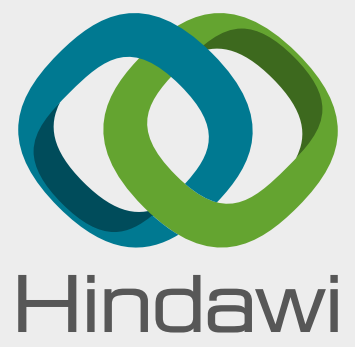

Submit your manuscripts at

www.hindawi.com
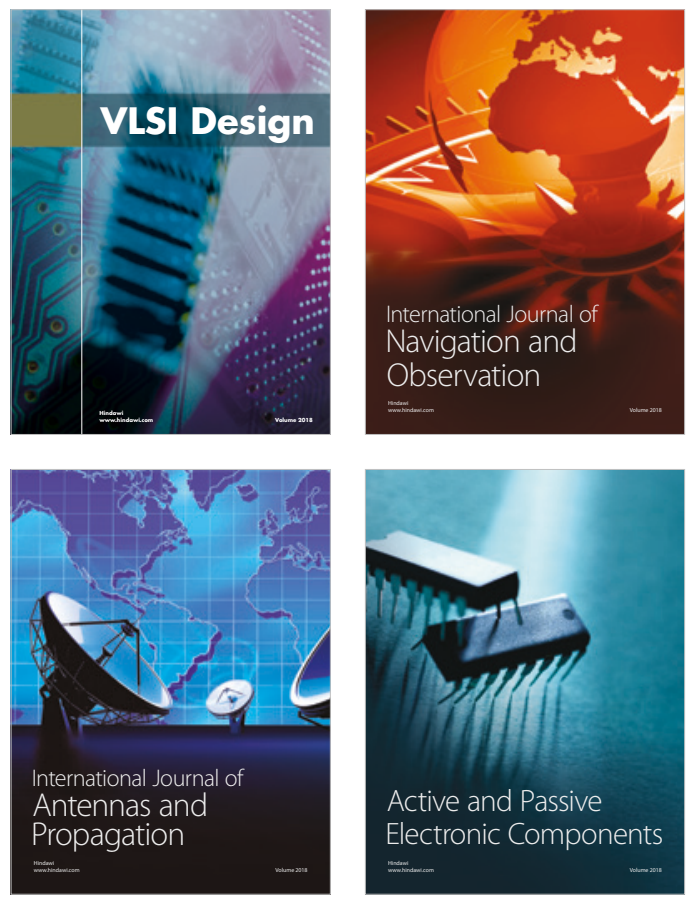
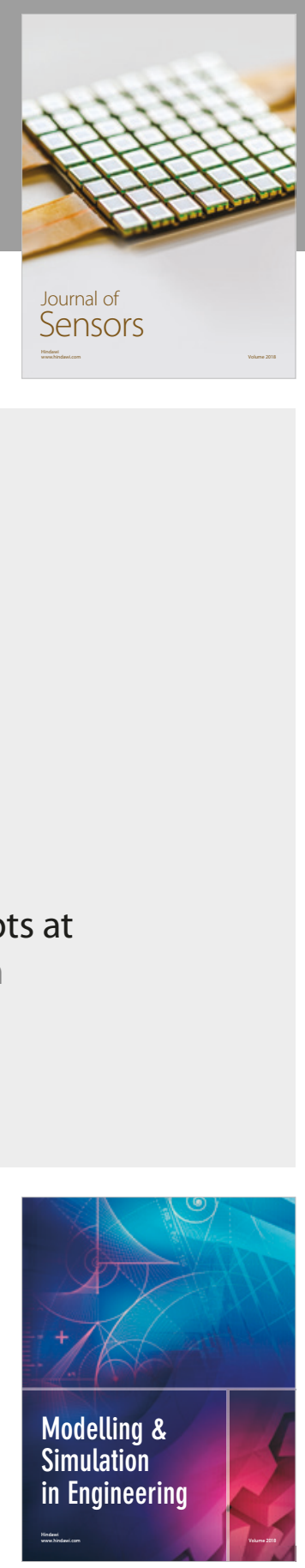

\section{Advances \\ Multimedia}
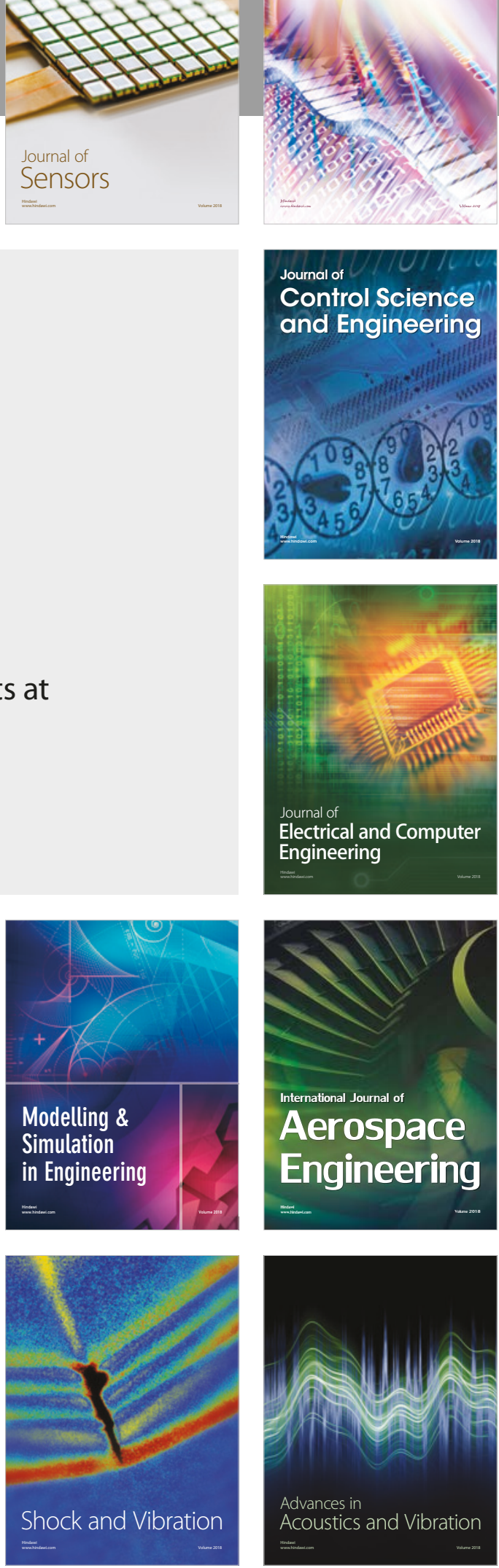